\title{
Effects of Long-Term Grafting on Follicular Growth in Porcine Ovarian Cortical Grafts Xenoplanted to Severe Combined Immunodeficient (SCID) Mice
}

\author{
Noriko KAGAWA ${ }^{1)}$, Yohichiro SAKURAI ${ }^{1)}$, Takashi MIYANO ${ }^{2)}$ and \\ Noboru MANABE ${ }^{3)}$ \\ 1) Unit of Anatomy and Cell Biology, Department of Animal Sciences, Kyoto University, Kyoto \\ 606-8502, ${ }^{2}$ Laboratory of Reproductive Biology and Biotechnology, Faculty of Agriculture, \\ Kobe University, Kobe 657-8501, ${ }^{3)}$ Research Unit of Animal Life Sciences, Animal Resource \\ Science Centre, Graduate School of Agricultural and Life Sciences, The University of Tokyo, \\ 3145 Ago, Ibaraki-Iwama 319-0206, Japan
}

\begin{abstract}
To establish a tool for the study of follicular growth and development, we xenotransplanted small pieces (approximately $1 \mathrm{~mm}^{3}$ ) of porcine ovarian cortical tissues containing only primordial follicles and small preantral follicles under the capsules of kidneys of severe combined immunodeficient (SCID) mice (8-10 weeks old). The changes in cell proliferation and cell death/ apoptosis, and vascularization in xenotransplanted follicles during follicular growth and development were analyzed histochemically at 1-26 weeks after operation. Follicles in grafted ovarian tissues grew rapidly forming an antral cavity (a hallmark of tertiary follicles) at 1 week after grafting. The diameter of the follicles in transplanted tissues ranged from 0.5 to $1.5 \mathrm{~mm}$, from 0.5 to 2.0 $\mathrm{mm}$ and from 0.5 to $3.0 \mathrm{~mm}$ at 1,2 and 26 weeks after the operation, respectively. Histological observation of ovarian tissues at 26 weeks after grafting revealed that all grafts had abundant capillary vessels, which invaded from murine organs and surrounded the growing follicles. Grafted small preantral follicles developed to the antral stages at 1 week after grafting and growing antral follicles survived at 26 weeks after grafting. The oocytes in the growing follicles were easily recovered for evaluating the quality. Our simple xenografting sysytem is easy to use and a good experimental tool for the study of folliclular growth and development in porcine ovaries.

Key words: Angiogenesis, Follicular growth and development, Porcine ovary, Severe combined immunodeficient (SCID) mouse, Xenotransplantation
\end{abstract}

(J. Reprod. Dev. 51: 77-85, 2005)

$\mathbf{T}$ he ovarian follicles are the fundamental structural and functional unit of the mammalian ovaries and consist of an oocyte, cumulus cells, granulosa cells, a basement membrane, and associated inner and outer theca cells arranged adjacent to the basement membrane. Normal/ healthy follicular growth and development are

Accepted for publication: October 4, 2004 Correspondence: N. Manabe

(e-mail: amanabe@mail.ecc.u-tokyo.ac.jp) essential for the maturation of an oocyte, that can undergo fertilization and develop to an embryo, and depend upon a complex sequence of cellular interactions within the follicles. The cross-talk and interaction among follicular cells create a unique and progressively shifting environment during the growth and development of the oocyte. During the oocyte's growth and development, less than $1 \%$ of follicles continue normal growing, with most of the rest undergoing atresia [1-4]. Recently, the selective 
mechanism in ovarian follicles, and the mechanism regulating follicular growth and development have been investigated using an in vitro culture system of ovarian tissues [5-9]. Several good models for studying follicular growth and development have been developed in rodents $[5,6]$, but no appropriate model in vitro has been established for humans and domestic animals [7-9]. Since follicles in humans and domestic animals have a comparatively great diameter and a thick basement membrane and thecal layer, the transportation of requisites for follicular growth and development might be restricted in conventional in vitro culture systems. On the other hand, when fresh ovarian tissues prepared from marmoset monkeys [10], cows [11], sheep [12], cats [13] and humans [14] were xenografted under the kidney capsules of immunodeficient animals, follicular growth and development were observed in these grafted ovarian tissues. It seems that the xenotransplant culture system has potential as an experimental tool for researching the growth and development of follicles and oocytes. For the xenoplant culture of ovarian tissues, athymic nude mice (lacking $\mathrm{T}$ lymphocytes) and/or severe combined immunodeficiency (SCID) mice (lacking B- or Tlymphocyte dependent immunity) have also been used [5-10].

In the present study, to establish an appropriate experimental tool for the study of follicular growth and development, we prepared cortical tissue slices from porcine ovaries containing many primordial follicles and preantral follicles, and then xenotransplanted them under the kidney capsules of SCID mice. To understand the relationship between follicular growth (cell proliferation) and atresia (cell death/apoptosis) in grafted tissues, the levels of cell proliferation and apoptosis were observed histochemically. Moreover, to find out the transportation levels of the requisites for follicular growth and development, we immunohistochemically determined the structure and distribution of capillary vessels around the follicles using a specific antibody against CD34 antigen (a specific antigen of endothelial cell membrane of mouse capillary vessels).

\section{Materials and Methods}

\section{Animals}

Female SCID mice (8-10 weeks of age) purchased from Clea Japan (Tokyo, Japan) were housed under conditions of controlled temperature $(24 \pm 2 \mathrm{C})$, lighting (12 h light: $12 \mathrm{~h}$ darkness) and humidity (80 $\pm 5 \%$ ). During the experiment, each animal cage was filter-topped (covered with a high-efficiency particulate air filter; Clea Japan), and animals had free access to sterilized food and bottled water. Upon arriving from the breeding suite, mice were allowed to acclimate for 1 week. They received humane care as outlined in the Guide for the Care and Use of Laboratory Animals (Kyoto University Animal Care Committee according to NIH No. 8623; revised 1999).

\section{Porcine tissue preparation and xenotransplantation}

Ovaries were obtained from prepubertal gilts slaughtered at a local abattoir and transported to the laboratory at $27-30 \mathrm{C}$ within $40 \mathrm{~min}$. Sliced tissues of ovarian cortex were prepared and placed immediately into sterile phosphate buffered saline (PBS; pH 7.4). Under a surgical dissecting microscope (SZ40; Olympus, Tokyo, Japan), the ovarian tissues were cut into small cubes (approximately $1 \mathrm{~mm}^{3}$ ) containing primordial, primary and secondary follicles, but no tertiary follicles, under sterile conditions, that is, all operations were performed in the cavity of a bioclean bench (Sanyo, Osaka, Japan). Female SCID mice were anesthetized with an intraperitoneal injection of sodium pentobarbital (Dainippon Pharmaceutical Co, Kyoto, Japan), and both kidneys were exteriorized. Cubes of ovarian tissues were inserted under the capsule space of each kidney using a hand-made glass pipette. Xenoplanted mice were kept for 1, 2 or 26 weeks (25 mice in each group).

\section{Histopathology and histochemistry}

To label the proliferating cells, 5-bromo-2'deoxyuridine (BrdU; $100 \mathrm{mg} / \mathrm{kg}$ body weight; Sigma Aldrich Chemicals, St. Louis, MO, USA) was injected into the peritoneal cavity $2 \mathrm{~h}$ before sacrifice.

For microscopic analysis of follicular growth and atresia, and angiogenesis, porcine ovarian tissues were recovered from the kidney of mice anesthetized with diethylether. The outward 
appearance of each follicle was recorded, and follicular diameters were measured using an ocular micrometer and verified subsequently from estimations of maximum follicular diameters in histological sections. The samples were fixed in $10 \%$ phosphate-buffered formalin $(\mathrm{pH} 7.4$; Wako Pure Chemicals, Osaka, Japan), dehydrated through a graded ethanol series and embedded in Histosec (Merck, Darmstadt, Germany). Serial sections $3 \mu \mathrm{m}$ thick were cut on a microtome, and mounted on glass slides precoated with 3-aminopropyltrimethoxysilane (silane; Sigma).

For conventional histopathological evaluation, one of the serial sections was stained with hematoxylin and eosin. All slides were mounted with Entellan (Merck), and examined by light microscope (BX51; Olympus).

Cells were confirmed to be proliferating using two immunohistochemical techniques: immunochemistry using the BrdU incorporated into the genomic DNA of proliferating cells; and immunostaining using a specific antibody against proliferating cell nuclear antigen (PCNA). Tissue sections were immunohistochemically stained for BrdU and PCNA. Briefly, they were dewaxed in xylene, rehydrated in descending concentrations of ethanol, and washed in distilled water. Endogeneous peroxidase activity was quenched with a 10 min incubation in $3 \%(v / v) \mathrm{H}_{2} \mathrm{O}_{2}$ in Trisbuffer saline (TBS; $0.05 \mathrm{M}$ Tris- $\mathrm{HCl}$ and $0.15 \mathrm{M}$ $\mathrm{NaCl}, \mathrm{pH}$ 7.6) at room temperature (22-25 C). After a wash in TBS, the sections were incubated with horseradish peroxide (HRP)-conjugated mouse monoclonal anti-BrdU antibody (1:200; Dako, Copenhagen, Denmark), or with HRPconjugated mouse monoclonal anti-PCNA antibody (1:200; Dako) for $1 \mathrm{~h}$ at room temperature, and then washed well with TBS. Positive cells were visualized by staining with liquid 3,3-diaminobenzidine (DAB) substrate (EnVision kit; Dako). The sections were counterstained with hematoxylin, washed with distilled water, dehydrated, mounted with Entellan and examined by light microscope. As negative controls, adjacent sections incubated without any antibody were prepared in each experimental run.

Apoptotic cells were identified histochemically by the terminal deoxy nucleodyl transferase (TdT)mediated biotinylated deoxyuridine triphosphate nick end-labeling (TUNEL) method. Adjoining sections from each specimen were stained by the
TUNEL method using a commercial kit (Apop Tag; Oncor Inc., Gaithersburg, MD, USA) to allow visualization of the apoptotic cells. Briefly, the sections were incubated with $20 \mu \mathrm{g} / \mathrm{ml}$ proteinase $\mathrm{K}$ (Sigma) in PBS for $15 \mathrm{~min}$ at room temperature, and then immersed in $2 \%(\mathrm{v} / \mathrm{v}) \mathrm{H}_{2} \mathrm{O}_{2}$ in methanol for $5 \mathrm{~min}$ to inhibit endogenous peroxidase activity. After preincubation with equilibration buffer for 10 $\mathrm{min}$ at $37 \mathrm{C}$, the sections were incubated with TdT solution containing $45 \mu \mathrm{M}$ ddATP and $5 \mu \mathrm{M}$ digoxigenin (DIG)-ddUTP for $1 \mathrm{~h}$ at $37 \mathrm{C}$, and then immersed in double strength salt sodium citrate buffer to stop the labeling reaction. After washing, the sections were incubated with HRP-labeled antiDIG antibody solution (Boehringer Mannheim, Indianapolis, IN, USA) for $30 \mathrm{~min}$ at room temperature and then reacted with $0.05 \%(\mathrm{w} / \mathrm{v})$ $\mathrm{DAB}$ and $0.002 \%(\mathrm{v} / \mathrm{v}) \mathrm{H}_{2} \mathrm{O}_{2}$ in $0.05 \mathrm{M}$ Tris- $\mathrm{HCl}$ ( $\mathrm{pH}$ 7.2) for 1-2 min at room temperature. After washing with distilled water, the sections were dehydrated through a graded ethanol series were mounted with Entellan and examined by light microscope. The following positive and negative controls were included in each experimental run. As negative controls, some sections were incubated without either TdT or DIG-ddUTP. As a positive control, tissue sections were treated with DNase I (1 $\mu \mathrm{g} / \mathrm{ml}$; Boehringer), $140 \mathrm{mM}$ sodium cacodylate, 4 $\mathrm{mM} \mathrm{MgCl} 2$ and $0.1 \mathrm{mM}$ dithiothreitol in $30 \mathrm{mM}$ Tris- $\mathrm{HCl}$ (pH. 7.2) for $10 \mathrm{~min}$ at room temperature before the exposure to TdT, and paraffin sections prepared from young adult rat testis were used as physiological positive controls.

Adjoining sections from each specimen were immunohistochemically stained for CD34 antigen. Briefly, the sections were dewaxed in xylene, rehydrated in descending concentrations of ethanol, and washed in distilled water. Endogeneous peroxidase activity was quenched with a 10 min incubation in $3 \%(v / v) \mathrm{H}_{2} \mathrm{O}_{2}$ in PBS at room temperature. Treatment with $5 \%(\mathrm{v} / \mathrm{v})$ fetal calf serum was follwed by incubation with mouse monoclonal anti-mouse CD34 antibody (1:200; Hycult Biotechnology, Uden, the Netherlands) for $18 \mathrm{~h}$ at $4 \mathrm{C}$. Sections were incubated with $100 \mu \mathrm{l} /$ section of goat anti-mouse-avidin-biotin-HRP complex solution (ABC-HRP; Dako) for $30 \mathrm{~min}$ at room temperature, washed in PBS and stained with liquid DAB substrate. The sections were counterstained with hematoxylin, washed with distilled water, dehydrated, mounted with 
Entellan, and examined by light microscope. As a negative control, adjoining sections incubated without any antibody were prepared in each experimental run.

\section{Follicular assessment}

According to Morbeck et al. [15], developmental stages of follicules were morphologically defined as follows: primordial (oocyte surrounded by a single flat layer of follicle epithelial cells / pre-granulose cells), primary (single layer of cuboidal granulosa cells), early secondary (2 to 4 layers of granulosa cells), late secondary follicule (more than 5 layers of granulosa cells, but no antrum) and tertiary (antral follicle; follicles containing an antral cavity, more than $0.5 \mathrm{~mm}$ in diameter). Moreover, each follicle was classified as either healthy, with a normalshaped cumulus-oocyte-complex (COC) surrounded by granulosa cells, an intact basement membrane, and granulosa cell nuclei without signs of pyknosis, or else atretic, i.e. not fulfilling these criteria.

\section{Results}

\section{Gross morphology of grafted ovaries}

Preantral small follicles (less than $0.1 \mathrm{~mm}$ in diameter; primordial follicles, primary follicles, early secondary follicles or late secondary follicles) in pre-grafted tissues of porcine ovaries grew rapidly forming an antral cavity (a hallmark of tertiary follicles) by 1 week after grafting. As shown in Fig. 1, the diameter of primary and secondary follicles in xenotransplanted ovarian tissues were less than $0.3 \mathrm{~mm}$, and that of tertiary follicles was more than $0.5 \mathrm{~mm}$. The diameter of the follicles was from 0.5 to $1.5 \mathrm{~mm}$, from 0.5 to 2.0 $\mathrm{mm}$ and from 0.5 to $3.0 \mathrm{~mm}$ at 1 (Fig. 1A), 2 and 26 (Fig. 1B) weeks after the operation, respectively. In most cases at 26 weeks after the operation, well growing antral follicles were observed on the surface area of the SCID mouse kidneys, remarkable neovascularization was seen on the surface of these antral follicles under a dissecting microscope. In some cases, a part of ovarian tissue adhered to the mouse liver.

\section{Histochemistry}

Conventional histopathology showed 3 small tertiary follicles in grafted ovarian tissue abutting the mouse kidney at 1 week after grafting (Fig. 2B and $\mathrm{E})$. The mouse kidneys were wrapped around the grafted ovarian tissues. After long-term grafting (26 weeks after the operation), abundant capillary vessels had infiltrated the ovarian tissues and surrounded the thecal layers of well-growing tertiary follicles (Fig. 2C and F). In the ovarian tissues with such large tertiary follicles, spontaneous luteinization of granulosa cells and infiltrated blood were seen, indicating the luteinizing process have
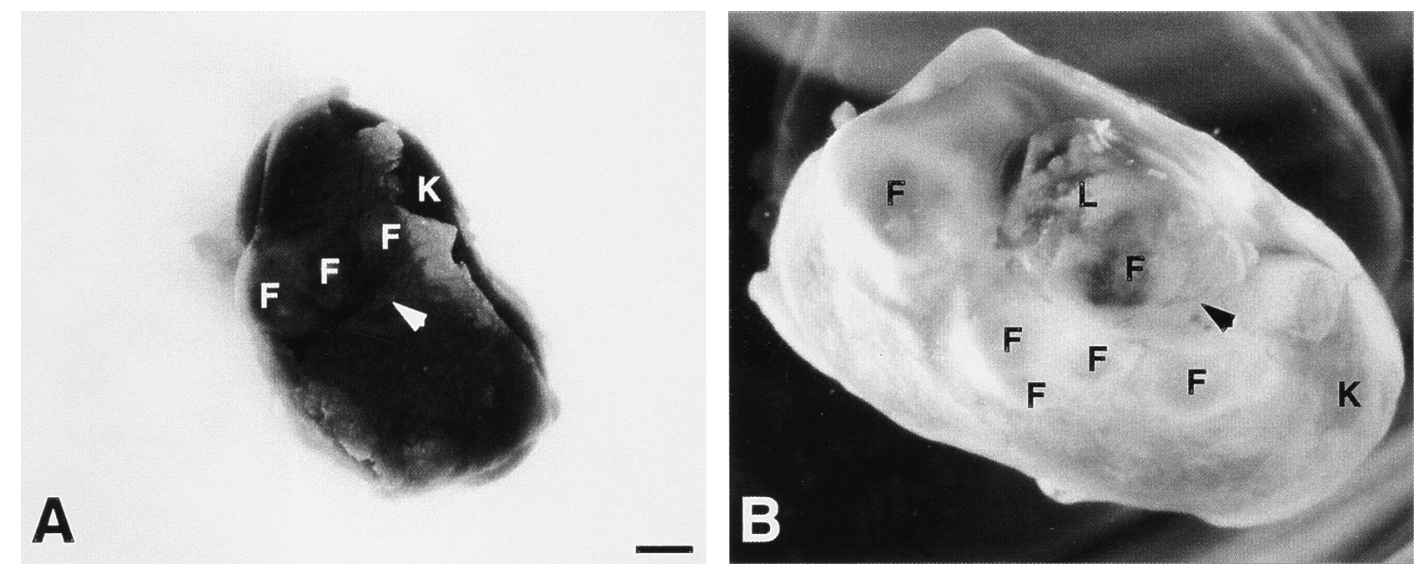

Fig. 1. Gross morphology of SCID mouse kidneys with xenotransplanted porcine ovarian tissues. Small pieces of ovarian tissues, in which no tertiary follicles exist, were transplanted under the capsules of SCID mouse kidneys $(\mathrm{K})$, and the kidneys were removed 1 and 26 weeks after the operation $(\mathrm{A}$ and $\mathrm{B}$, respectively). Tertiary follicles (F; more than $1.5 \mathrm{~mm}$ in diameter) can be seen. Remarkable neovascularization (arrowheads) can be observed in the transplanted area of the mouse kidneys. A part of ovarian tissue adheres to the mouse liver (L). Bar: $1 \mathrm{~mm}$. 

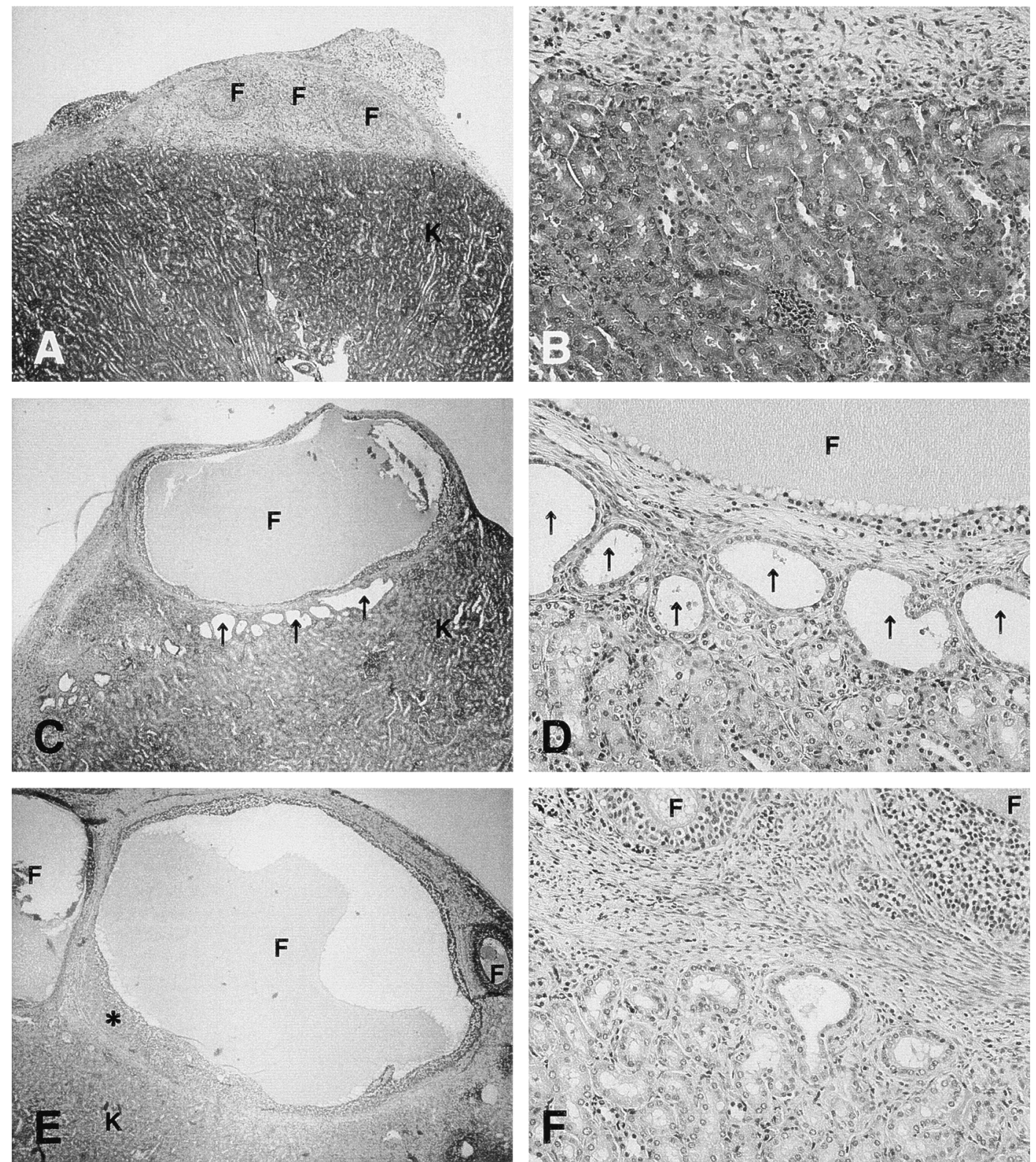

Fig. 2. Histological section of grafted ovarian tissues and SCID mouse kidneys (K). Tertiary follicles developed 1 (A and B), 2 (C and D) and 26 (E and F) weeks after operation. The mouse kidneys were wrapped around the grafted ovarian tissues. Expanded renal tubules can be seen (arrows in C and D), and spontaneous luteinization of granulosa cells (asterisk in E) and infiltrated blood cells occurred in the tertiary follicles after long-term culture (E). Magnification: A, C and E: $\times 200$, and B, D and F: $\times 400$.

been proceeding.

Abundant proliferating cells (PCNA-positive cells and BrdU-positive cells) were detected in the stromal area, theca internal layer and theca external layer of the porcine ovarian tissues at 1 week after the operation (Fig. 3A), but few proliferating cells were found at 26 weeks after xenoplantation (Fig. 3B). Few apoptotic cells (TUNEL-positive cells) were observed in the grafted tissues (data not shown), indicating that the follicular atretic process may be blocked in the xenoplanted ovarian tissues.

Remarkable neovascularization was immuno- 

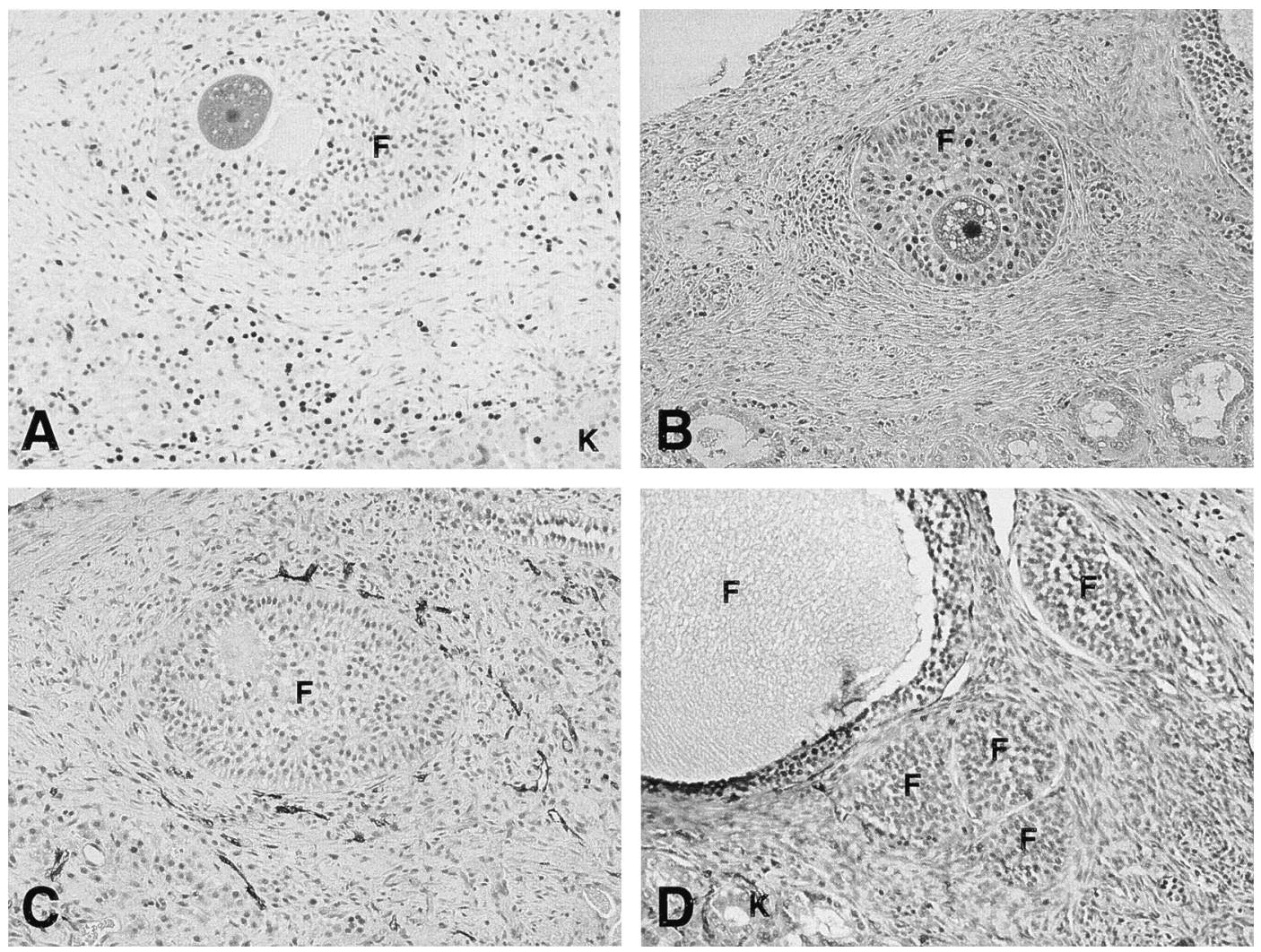

Fig. 3. Immunohistchemical staining for PCNA (A and B) and CD34 (C and D), which are marker proteins for proliferating cells and murine endothelial cells of capillary vessels, respectively. Abundant PCNApositive proliferating cells were demonstrated in the granulosa cell layers and thecal layers of follicles and ovarian stromal area at 1 week after the transplantation (A). At 26 weeks after the operation, an increased number of proliferating cells in granulosa cell layers were observed, but few proliferating cells were noted in thecal layers or stromal cell areas of the grafts (B). At both 1 and 26 weeks after the operation (C and D, respectively), CD24-positive staining, indicating the existence of capillary vessel networks, was observed in the thecal area, in which well organized vessel networks were developed.

Magnification: $\times 200$.

histochemically demonstrated at 1 week after the operation (Fig. 3C). The capillary vessels were expanded from mouse renal tissues, well developed and wrapped around the tertiary follicles but not small preantral follicles. The capillary vessel network localized to the thecal layer area (Fig. 3D). As describe above, well growing antral follicles (more than $3 \mathrm{~mm}$ in diameter) were observed on the kidney's surface in the SCID mice after long-term grafting (26 weeks after the grafting), and abundant neovascularization was evident on the surface of such antral follicles under a dissecting microscope. Immunohistochemical observation confirmed the remarkable neovascularization in the thecal layer area of transplanted ovarian tissues. Such tertiary follicles with large antral cavities contained oocytes that remained at the germinal vesicle (GV) stage (Fig. 3D). In some cases at 26 weeks after the operation, expanded renal tubules (cystic renal tubular dilation) invaded extremely large antral follicles, and antral cavities were filled with abundant erythrocytes and macrophages. Moreover, in a few large tertiary follicles, spontaneous luteinization of granulosa cells was seen.

\section{Discussion}

Successful results have been reported for xenotransplantation experiments using mouse 
ovarian tissues, which were grafted in SCID mouse kidneys [5, 6], but no acceptable data have been obtained for farm animals. Since, compared with the mouse, the oocytes and follicles of large domestic animals have a relatively longer growth phase and are extremely large, examinations using the ovarian tissues of domestic animals are quite challenging. Porcine oocytes grow from $30 \mu \mathrm{m}$ (not including the zona pellucida) to $120-125 \mu \mathrm{m}$ in diameter during the primordial folliclestage and grow to the final preovulatory stage in a period of 3 months [16]. In cattle, the period of oocyte maturation is 6 months [17]. These physiological time schedules imply that long-term grafting systems for farm animals have to be established for the utilization of primordial follicles by xenografting. However, acceptable culture systems supporting the entire developmental course in larger animals have to be developed. In the present study, we examined the effects of long-term grafting (26 weeks) of porcine ovaries xenotransplanted under the kidney capsules of female SCID mice. At 26 weeks after the transplantation of small pieces of pig ovaries, large tertiary follicles in which large antral cavities were developed were observed on the surface area of the kidney in SCID mice. The mouse kidneys were wrapped around the porcine ovarian tissues, and expanded renal tubules were observed. In a few large follicles, abundant erythrocytes and macrophages invaded the antral cavities, and spontaneous luteinization of granulosa cells occurred. Long-term in vivo organ culture by xenotransplantation is different from in vitro organ culture. The long-term culture may be involved in the aging/senescence of recipient SCID mice and pressure for the chorionic membrane of the renal capsule, in which grafted follicles grow and develop. Recently, Kaneko et al. [18] reported that the fertilization of oocytes from porcine primordial follicles in neonatal ovarian tissues could be achieved by a combination of xenografting and in vitro culture. They optimized the timing of equine chorionic gonadotropin (eCG) treatment of the host mice to improve the growth of antral follicles in the grafted tissues, and then combined the methods with in vitro culture for oocytes. However, the growth and development of primordial follicles in the xenografted tissues prepared from adult porcine ovaries were not good enough. The present study showed that the growth and development of preantral follicles could be achieved by long-term xenografting culture.

Xenotransplantation of small oocytes to nude or SCID mice may be a substitute for an effective longterm culture system. Mice homozygous for the SCID mutation lack both humoral and cellmediated immunity due to the absence of mature $\mathrm{T}$ - and B-lymphocytes [17]. In addition to having a healthy life span and tolerating surgery well in a sterile environment, SCID mice readily accept ovarian tissues from other mammalian species. Gosden et al. [19] developed xenotransplantation of mammalian follicles into SCID mice as a model for investigating the early stage of follicular development and for verifying the viability of follicles after cryopreservation. When cortical slices of cat or sheep ovaries were xenotransplantated under the kidney capsules of SCID mice, some follicles reached a diameter of $3.0 \mathrm{~mm}$ with a normal antrum forming after a few months $[13,20]$. In human ovarian grafts, the antral stage follicles recovered after 17 weeks of follicle stimulating hormone (FSH) treatment [21]. However, there have been no reports of success in the full maturation, fertilization, or birth from xenotransplantated oocytes in large mammalian species.

Previous studies showed that porcine antral follicles produce substantial amounts of angiogenic factor, ex. vascular endothelial growth factor (VEGF), during their growth and response to gonadotropic stimulation [20]. In the follicular wall area, granulosa cells represent the major source of the angiogenic factor produced in response to $\mathrm{FSH}$, and secreted VEGF tends to accumulate in the antral cavity, where it reaches levels as high as 20 $\mathrm{ng} / \mathrm{ml}$. This store and the resulting diffusion create an angiogenic gradient that is likely to regulate the development of the capillary vessel network and its architecture within the follicle wall. We immunohistochemically demonstrated the neovascularization around the follicular walls of large tertiary follicles in grafted tissues. New microvessels remarkably expanded at 1 week after the operation, and then these microvessels invaded the grafted tissues and localized in the theca-layer area at 2 weeks after grafting. In some case, small capillaries passed through the primary follicle compartment. In tertiary follicles on shortterm grafting ( 2 weeks), abundant capillary vessel network developed in the thecal layer area. For 
appropriate growth and development of the follicles, efficient compensatory mechanisms, an adequate vascular network to meet the increasing needs of growing follicles, is required. A proper blood supply may be a major regulating factor that controls the overall follicle cell function as well as oocyte growth and maturation. Our data imply that only follicles which are surrounded by the murine capillary vessel network system can grow and develop in xenotransplantated ovarian tissues. In this context, the ability of a follicle to produce VEGF may represent an essential, limiting factor for growth and, as such, be a distinguishing characteristic of follicles that have overcome selection and entered the follicular pool.

In the present study, we demonstrated that a good number of tertiary follicles in xenotransplanted cortical tissues of porcine ovaries could be recovered in SCID mice 2 and 26 weeks after grafting. The long-term culture system with xenografting is considered useful for studying the correlation of oocyte growth and maturation with follicular development. Moreover, the combination of xenotransplantation and in vitro fertilization may be an even more powerful tool for investigating the basic mechanisms of oocyte growth and maturation, and of follicular development. Thus, our results have resolved a major limitation to xenotransplantation as a model of in vivo culture, in that we were able to obtain large tertiary follicles from early preantral follicles in porcine ovarian tissues. Subsequent in vitro maturation resulted in progress to the metaphase I stage of oocytes (data not shown) from short-term grafting ( 1 and 2 weeks) but not long-term grafting in SCID mice. Therefore, we suggest that for practical use (ex. in vivo culture for the cryopreserved ovarian tissues to obtain newborns), in vivo short-term grafting would be more suitable than long-term grafting, and that in vivo long-term grafting would be useful for revealing molecular mechanisms in fundamental research on oocyte growth and maturation, and of follicular growth and atresia.

\section{Acknowledgements}

The study was supported by a Grant-in-Aid for Creative Scientific Research (13GS0008) from the Ministry of Education, Culture, Sports, Science and Technology of Japan to N. M. and by a Grant-inAid for Scientific Research (S) (16108003) from the Japan Society for The Promotion of Science to N. M.

\section{References}

1. Fortune JE. Ovarian follicular growth and development in mammals. Biol Reprod 1994; 50: 225-232.

2. Manabe, N, Kimura Y, Myoumoto A, Matsushita H, Tajima C, Sugimoto M, Miyamoto H. Role of granulosa cell apoptosis in ovarian follicle atresia. In: Yamada T, Hashimoyto Y (eds.), Apoptosis: Its Roles and Mechanism. Tokyo: Academic Societies Japan; 1998: 97-111.

3. Manabe N, Inoue N, Miyano T, Sakamaki K, Sugimoto $\mathbf{M}$, Miyamoto $\mathbf{H}$. Ovarian follicle selection in mammalian ovaries: regulatory mechanisms of granulosa cell apoptosis during follicular atresia. In: Leung PK, Adashi E (eds.), The Ovary, 2nd edition. Amsterdam: Academic Press / Elsevier Science Publishers; 2003: 369-385.

4. Evans ACO. Characteristics of ovarian follicle development in domestic animals. Reprod Dom Anim 2003; 38: 240-246.

5. Eppig JJ, Schroeder AC. Capacity of mouse oocytes from preantral follicles to undergo embryogenesis and development to live young after growth, maturation, and fertilization in vitro. Biol Reprod
1989; 41: 268-276.

6. Eppig JJ, O’brien MJ. Development in vitro of mouse oocytes from primordial follicles. Biol Reprod 1996; 54: 197-207.

7. Hirao Y, Nagai T, Kubo M, Miyano T, Miyake M, Kato S. In vitro growth and maturation of pig oocytes. J Reprod Fertil 1994; 100: 333-339.

8. Wu J, Zhang L, Liu P. A new source of human oocytes: preliminary report on the identification and maturation of human preantral follicles from follicular aspirates. Hum Reprod 1998; 13: 2561-2563.

9. Cecconi S, Barboni B, Coccia M, Mattioli M. In vitro development of sheep preantral follicles. Biol Reprod 1999; 60: 594-601.

10. Candy CJ, Wood MJ, Whittingham DG. Follicular development in cryopreserved marmoset ovarian tissue after transplantation. Hum Reprod 1995; 10: 2334-2338.

11. Senbon S, Ota A, Tachibana M, Miyano T. Bovine oocytes in secondary follicles grow and acquire the meiotic competence in severe combined immunodeficient mice. Zygote 2003; 11: 159-165. 
12. Gosden RG, Baird DT, Wade JC, Webb R. Restoration of fertility to oophorectomized sheep by ovarian autografts stored at $-196^{\circ} \mathrm{C}$. Hum Reprod 1994; 9: 597-603.

13. Oktay K, Newton H, Mullan J, Gosden RG. Development of human primodial follicles to antral stages in SCID/hpg mice stimulated with follicle stimulating hormone. Hum Reprod 1998; 13: 11331138.

14. Wu J, Emery BR, Carrell DT. In vitro growth, maturation, fertilization, and embryonic development of oocytes from porcine preantral follicles. Biol Reprod 2001; 64: 375-381.

15. Morbeck DE, Esbenshade KL, Flowers WL, Britt JH. Kinetics of follicle growth in the prepubatal gilt. Biol Reprod 1992; 47: 485-491.

16. Lussier JG, Matton P, Dufour JJ. Growth rates of follicles in the ovary of the cow. J Reprod Ferti 1987; 81: 301-307.
17. Bosma GC, Custer RP, Bosma MJ. A severe combined immunodeficiency mutation in the mouse. Nature 1983; 301: 527-530.

18. Kaneko H, Kikuchi K, Noguchi J, Hosoe M, Akita T. Maturation and fertilization of porcine oocytes from primordial follicles by a combination of xenografting and in vitro culture. Biol Reprod 2003; 69: 1488-1493.

19. Gosden RG, Boulton MI, Grant K, Webb R. Follicular development from ovarian xenografts in SCID mice. J Reprod Fertil 1994; 101: 619-623.

20. Oktay K, Newton H, Aubard Y, Salha O, Gosden RG. Cryopreservation of immature human oocytes and ovarian tissue: an emerging technology? Fertil Steril 1998; 69: 1-7.

21. Barboni B, Turriani M, Galeati G, Spinaci M, Bacci ML, Forni M, Mattioli M. Vascular endotherial growth factor production in growing pig antral follicles. Biol Reprod 2000; 63: 858-864. 